\title{
Avaliação da reprodutibilidade do método de determinação da maturação esquelética por meio das vértebras cervicais
}

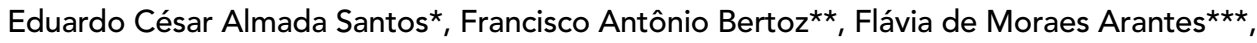

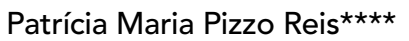

\begin{abstract}
Resumo
Dentre os diversos meios de se determinar o grau de maturação esquelética do paciente destaca-se o Método de Lamparski modificado por Hassel e Farman, em 1995, que propuseram a identificação do estágio da maturação por meio das modificações anatômicas das 2 as , 3 as e 4 as vértebras cervicais. Cientes das qualidades advogadas ao método citado surgiu o interesse em avaliar sua reprodutibilidade com o intuito de divulgá-lo e incorporá-lo como um elemento no diagnóstico e auxiliar no prognóstico dos tratamentos das más oclusões. A amostra constou de 100 telerradiografias em norma lateral de pacientes triados para tratamento ortodôntico na Faculdade de Odontologia de Araçatuba - UNESP nos períodos de 2000 e 2001. Foram incluídos pacientes de ambos os gêneros na faixa etária de 6 a 16 anos e a média de 9 anos e 7 meses. Três examinadores devidamente calibrados realizaram a avaliação das radiografias classificando-as em escores de 1 a 6. Após a análise dos resultados, os mesmos foram tabulados e submetidos ao coeficiente Kappa de concordância para avaliação inter e intra-examinador concluindo, dessa forma, a reprodutibilidade do referido método. O método de determinação da maturação esquelética por meio das vértebras cervicais mostrou-se reproduzível na avaliação do estágio em que o indivíduo se encontra na curva de crescimento.
\end{abstract}

Palavras-chave: Idade esquelética. Indicadores de maturação esquelética. Telerradiografia lateral. Vértebras cervicais. Crescimento craniofacial.

\section{INTRODUÇÃO}

A Ortodontia contemporânea vem evoluindo muito com o passar dos anos, tornando-se cada vez mais eficiente em relação ao diagnóstico, tratamento, previsibilidade e estabilidade dos resultados. Mercadanti ${ }^{14}$ relatou que dois terços dos casos tratados ortodonticamente incluem tipos de más oclusões onde o crescimento e desenvolvimento desempenham papel preponderante no êxito ou no fracasso da mecanoterapia. Em decorrência disto, a época ideal para iniciar o tratamento ortodôntico é considerada uma das causas de

* Professor Assistente Doutor da Disciplina de Ortodontia do Departamento de Odontologia Infantil e Social da Faculdade de Odontologia de Araçatuba - UNESP.

** Professor Titular Doutor da Disciplina de Ortodontia do Departamento de Odontologia Infantil e Social da Faculdade de Odontologia de Araçatuba - UNESP.

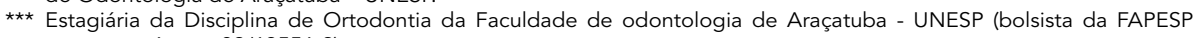
processo número 02/13556-2).

**** Mestra em Odontologia, Área de Concentração Ortodontia da Faculdade de Odontologia de Araçatuba - UNESP. 
controvérsias na literatura ${ }^{19,21,22}$. Os artigos recentes refletem o debate e oferecem opiniões distintas acerca do tratamento precoce ${ }^{21,22}$, realizado em duas fases (Ortopedia e Ortodontia Corretiva) versus o tratamento tardio, realizado em uma única fase (Ortodontia corretiva). O momento adequado para o início do tratamento precoce apresenta suma importância para a obtenção de resultados satisfatórios, considerando o período de crescimento pré-pubertário a melhor época para iniciar o tratamento ortopédico de más oclusões esqueléticas, pois durante esta fase ocorrem as alterações craniofaciais favorecendo uma coordenação destas com os procedimentos ortopédico-ortodônticos. O tratamento ortopédico tem como objetivo o aproveitamento do crescimento craniofacial do paciente direcionando-o para uma posição mais favorável. Embora seja de consenso geral a impossibilidade de estimular ou inibir o crescimento craniofacial além do potencial genético, a detecção da época de ocorrência do pico de crescimento favorece a eliminação de possiveis interferências ${ }^{14}$ pelo uso de aparelhos e técnicas adequadas, direcionando e favorecendo as relações dentoesqueléticas. As decisões relativas ao uso de aparelhos funcionais, tratamento ortodôntico com ou sem extrações e época da cirurgia ortognática são baseadas principalmente em ponderações relativas ao referido estágio de maturidade esquelética do paciente.

Existem diversos meios de se determinar o estágio em que o indivíduo se encontra na curva de crescimento, sendo eles a idade cronológica, idade dentária, idade circumpuberal que leva em consideração o aparecimento das características sexuais secundárias, a relação altura-peso e a idade esquelética. A idade esquelética representa o meio mais fidedigno e o mais utilizado atualmente ${ }^{8,17,18}$ uma vez que os demais apresentam grandes desvios comparados à média padrão e variações de tempo, duração e velocidade de indivíduo para indivíduo. Estes desvios e variações decorrem de fatores genéticos e raciais, condições climáticas, circuns- tâncias nutricionais, condições sócio-econômicas, alterações de uma maturação cada vez mais precoce do homem através do tempo (alterações seculares) e fatores hormonais e sexuais ${ }^{4,15,23}$. Identificase que as pessoas do gênero feminino antecedem dois anos em média o pico de crescimento puberal em relação às do gênero masculino ${ }^{14,17,18}$.

Alguns métodos são utilizados para determinação da maturação esquelética como o uso de radiografias carpais representadas mais especificamente pelo método de Greulich e Pyle ${ }^{6}$ e o método de Fishman ${ }^{4}$. Utiliza-se também a avaliação de radiografias periapicais do dedo polegar ${ }^{20}$ e a avaliação por meio das vértebras cervicais exemplificada pelos métodos de Lamparski ${ }^{11}$ e de Hassel e Farman ${ }^{9}$. Embora a radiografia carpal seja comprovadamente eficaz e segura, atualmente novas propostas para avaliação da idade óssea vêm surgindo com o intuito de reduzir o número de exposições radiográficas a que são submetidos os pacientes. Posto isto, esforços têm sido empregados no sentido de se utilizarem radiografias que fazem parte da documentação ortodôntica de rotina, por exemplo, as telerradiografias em norma lateral e as radiografias panorâmicas. Além de zelar pela saúde dos pacientes, esse procedimento que utiliza exames radiográficos de rotina simplifica a avaliação e reduz o custo para o paciente.

Com base nos dados apresentados anteriormente, propusemo-nos, neste trabalho, a avaliar a eficiência da reprodutibilidade do método de determinação da maturação esquelética por meio da visualização da imagem radiográfica das vértebras cervicais em 100 telerradiografias em norma lateral.

\section{MATERIAL E MÉTODO Material}

A amostra da pesquisa constou de 100 telerradiografias em norma lateral de pacientes triados para tratamento ortodôntico na Faculdade de Odontologia de Araçatuba - UNESP, nos períodos de 2000 e 2001. Convém salientar que foram incluídos pacientes de ambos os gêneros, com idades 
variando de 6 a 16 anos e a média de 9 anos e 7 meses. As telerradiografias em norma lateral foram realizadas obedecendo aos critérios técnicos de obtenção e revelação de filmes radiográficos ${ }^{15}$ preconizados pela Disciplina de Radiologia da Faculdade de Odontologia de Araçatuba - UNESP.

\section{Método}

As telerradiografias foram distribuídas aleatoriamente em envelopes enumerados de 1 a 100 sendo vedada a identificação, idade e elementos dentários dos pacientes com papel cartão preto procurando-se evitar a influência desses fatores sobre os examinadores durante a avaliação dos estágios de maturação esquelética (Fig. 1).

O método utilizado para determinação da maturação esquelética por meio das vértebras cervicais presentes nas telerradiografias em norma lateral foi o de Hassel e Farman ${ }^{9}$, modificado a partir do estudo de Lamparski ${ }^{11}$.

A avaliação das telerradiografias em norma lateral foi realizada por três examinadores sendo um aluno do $8^{\circ}$ período do curso de graduação em Odontologia, um mestrando na área de concentração em Ortodontia e um docente da disciplina de Ortodontia da FOA - UNESP. Os examinadores, antes de iniciarem a avaliação radiográfica, esclareceram-se a respeito do método selecionado e avaliaram dez telerradiografias não pertencentes à amostra em questão, com o objetivo de assimilar e padronizar o método. Com o intuito de verificar a confiabilidade da análise de cada examinador, os mesmos, após a calibração realizaram a avaliação das telerradiografias em um momento inicial, designado Tl. Após um período de quinze dias, tempo suficiente para que os examinadores não se recordassem das marcações realizadas na avaliação inicial, cada examinador reavaliou todas as radiografias denominando-as T2.

Para tanto, as radiografias foram analisadas individualmente pelos examinadores sobre um negatoscópio numa sala obscurecida, a fim de evitar a influência de qualquer informação na decisão do

\begin{tabular}{|c|c|}
\hline Variação numérica do coeficiente & Nível de concordância \\
\hline$<0.00$ & Pobre \\
\hline $0.00-0.20$ & Fraco \\
\hline $0.21-0.40$ & Razoável \\
\hline $0.41-0.60$ & Moderado \\
\hline $0.61-0.80$ & Substancial \\
\hline $0.81-1.00$ & Quase perfeito \\
\hline
\end{tabular}

resultado. Os dados obtidos, então anotados em uma folha seqüencial de 1 a 100 , foram tabulados e submetidos ao coeficiente Kappa de concordância para avaliação intra e interexaminadores. Segundo Landis e Koch ${ }^{12}$, o nível de concordância varia de 0 a 1 (Tab. 1).

\section{Método de avaliação da maturidade esquelética Método de Hassel e Farman?}

O método de Hassel e Farman ${ }^{9}$, modificado a partir do estudo de Lamparski ${ }^{11}$, consiste na observação das alterações anatômicas das 2 as, 3 as e 4 as vértebras cervicais $(\mathrm{C} 2, \mathrm{C} 3$ e $\mathrm{C} 4)$ presentes nas telerradiografias em norma lateral. A viabilidade deste método reside na fácil visualização dessas estruturas nas telerradiografias laterais, bem como o uso do colar protetor de tireóide não prejudicar a imagem radiográfica. Além disso, o ortodontista que se utiliza deste método e conhece a anatomia e morfologia das vértebras cervicais, torna-se apto a detectar possíveis anomalias nas mesmas como: fraturas, poliartrites, anquiloses, problemas degenerativos e defeitos congênitos ${ }^{2}$. Muitas anormalidades nas vértebras cervicais não se manifestam sintomaticamente até a adolescência, beneficiando um diagnóstico precoce e minimizando possíveis conseqüências severas ${ }^{9}$.

Neste método, enumera-se seis estágios de maturação baseados nas alterações morfológicas das vértebras cervicais denominados Indicadores de Maturação das Vértebras Cervicais (IMVCs): Iniciação, Aceleração, Transição, Desaceleração, 


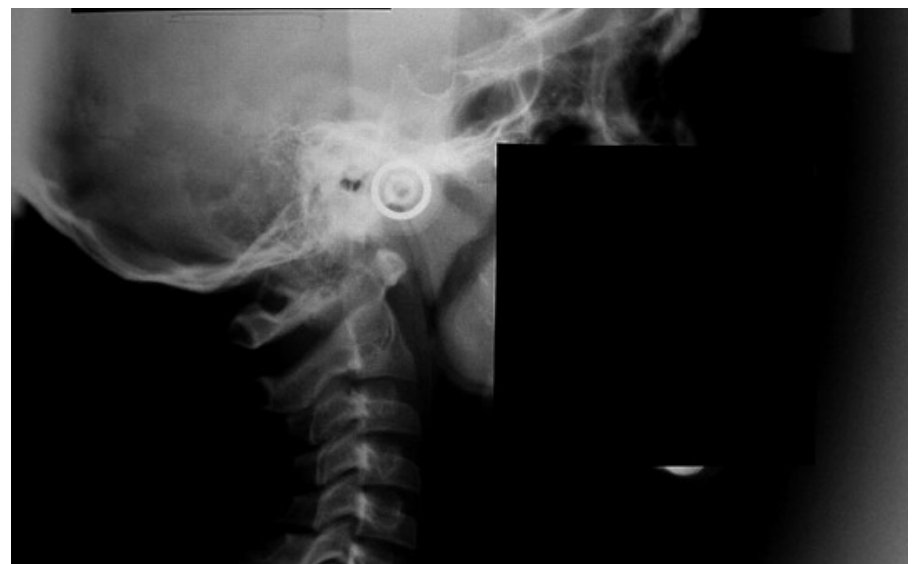

FIGURA 1 - Telerradiografia em norma lateral com o vedamento da identificação, idade e elementos dentários do paciente.

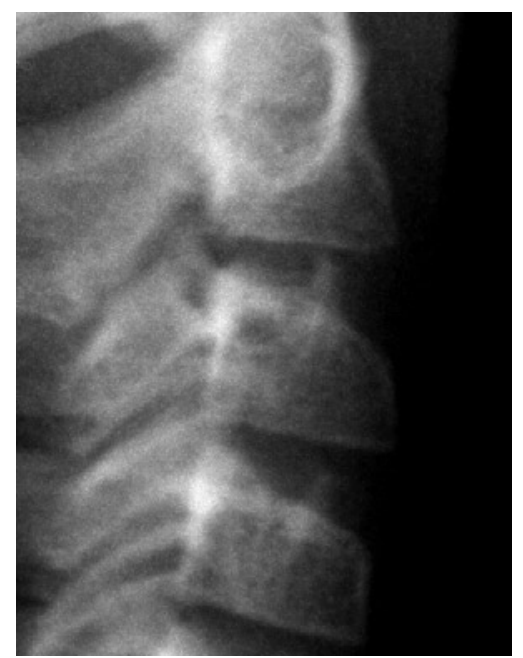

FIGURA 2 - Visualização das 2aㅗ $3^{\text {as }}$ e $4^{\text {as }}$ vértebras cervicais na fase de INICIAÇÃO.

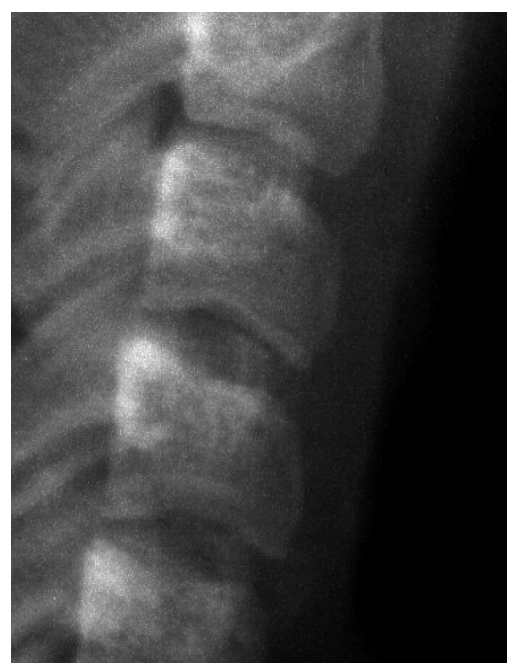

FIGURA 5 - Visualização das 2 ass, 3 as e $4^{\text {as }}$ vértebras cervicais na fase de DESACELERAÇÃO.

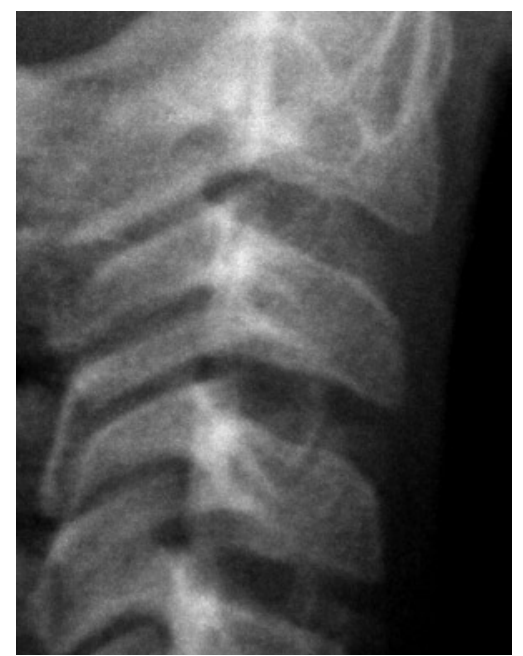

FIGURA 3 - Visualização das 2as, 3 as e 4 as vértebras cervicais na fase de ACELERAÇÃO.

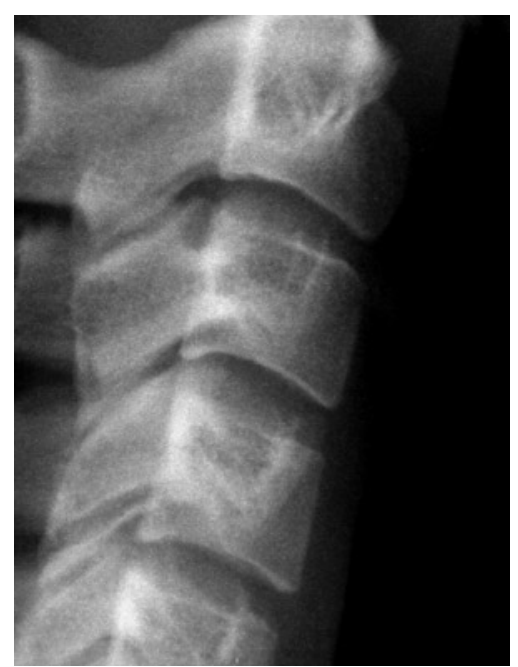

FIGURA 6 - Visualização das 2로 $3^{\text {as }}$ e $4^{\text {as }}$ vértebras cervicais na fase de MATURAÇÃO.

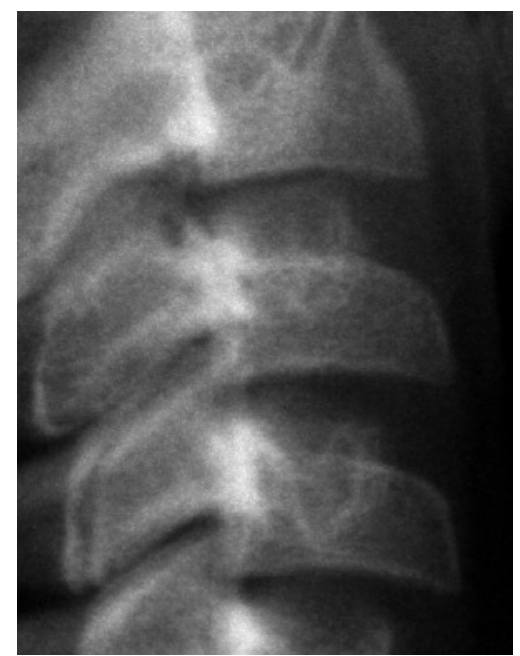

FIGURA 4 - Visualização das 2aㅗ $3^{\text {as }}$ e $4^{\text {as }}$ vértebras cervicais na fase de TRANSIÇÃO.

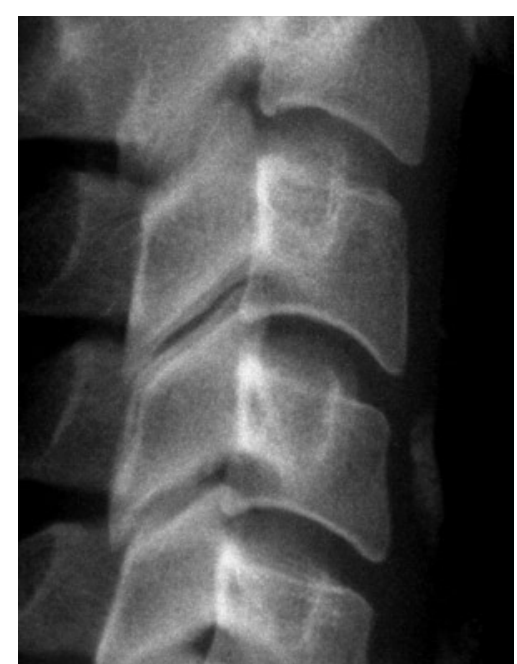

FIGURA 7 - Visualização das 2aㅗ $3^{\text {as }}$ e $4^{\text {as }}$ vértebras cervicais na fase de FINALIZAÇÃO. 
Maturação e Finalização. Nas figuras 2 a 7 pode-se observar as características morfológicas das vértebras cervicais de cada uma das seis fases propostas por este método e sua correlação com o crescimento puberal.

\section{1) Iniciação (Fig. 2)}

- Bordas inferiores das vértebras C2, C3 e C4 planas;

- Bordas superiores de C3 e C4 afuniladas de posterior para anterior;

- Expectativa de grande quantidade de crescimento puberal $(80 \%$ a $100 \%)$.

\section{2) Aceleração (Fig. 3)}

- Início do desenvolvimento de concavidades nas bordas inferiores de $\mathrm{C} 2$ e $\mathrm{C} 3$ e borda inferior da C4 plana;

- C3 e C4 com formatos tendendo a retangulares;

- Expectativa de crescimento puberal significante $(65 \%$ a $85 \%)$.

\section{3) Transição (Fig. 4)}

- Presença de concavidades evidentes nas bordas inferiores da $\mathrm{C} 2$ e C3;

- Início do desenvolvimento de uma concavidade na borda inferior da $\mathrm{C} 4$;

- C3 e C4 apresentam-se retangulares;

- Expectativa moderada de crescimento puberal $(25 \%$ a $65 \%)$.

\section{4) Desaceleração (Fig. 5)}

- Presença de concavidades evidentes nas bordas inferiores da C2, C3 e C4;

- Formato da C3 e C4 aproximando-se de um quadrado;

- Expectativa reduzida de crescimento puberal $(10 \%$ a $25 \%)$.

\section{5) Maturação (Fig. 6)}

- Presença de concavidades acentuadas nas bordas inferiores de $\mathrm{C} 2$, $\mathrm{C} 3$ e C4;
- Formato quadrado das vértebras C3 e C4;

- Expectativa de quantidade insignificante de crescimento puberal (5\% a $10 \%)$.

\section{6) Finalização (Fig. 7)}

- Presença de concavidades profundas nas bordas inferires de $\mathrm{C} 2$, C3 e C4;

- Altura das vértebras C3 e C4 ultrapassando sua largura;

- Crescimento puberal completo nesta fase.

\section{RESULTADOS}

A tabela 2 refere-se aos coeficientes de correlação derivados da comparação dos resultados da primeira e segunda avaliação dos mesmos examinadores, ou seja, do teste intraexaminador. Os resultados intraexaminadores demonstraram um nível de concordância de substancial (examinadores A e C) a quase perfeito (examinador B).

Já a tabela 3 revela o coeficiente de correlação advindos da comparação dos resultados entre os diferentes examinadores, ou melhor, do teste interexaminador. Os valores denotaram um nível de concordância substancial para todas as comparações. Os coeficientes de concordância altos indicam a possibilidade de se reproduzir o método de avaliação, tanto por um mesmo examinador, quanto pelos diferentes examinadores.

\begin{tabular}{|c|c|c|}
\hline \multicolumn{3}{|c|}{$\begin{array}{c}\text { Tabela 2 - Coeficiente Kappa de concordância para } \\
\text { avaliação do teste intra-examinador. }\end{array}$} \\
\hline Concordância & Porcentagem (\%) & Coeficiente Kappa \\
\hline A1 x A2 & 80 & 0.71 \\
\hline B1 x B2 & 95 & 0.91 \\
\hline C1 x C2 & 77 & 0.65 \\
\hline
\end{tabular}

\begin{tabular}{|c|c|c|}
\hline Concordância & Porcentagem (\%) & Coeficiente Kappa \\
\hline$A \times B$ & 76 & 0.61 \\
\hline$A \times C$ & 76 & 0.64 \\
\hline $\mathrm{B} \times \mathrm{C}$ & 75 & 0.60 \\
\hline
\end{tabular}


O nível de concordância maior na avaliação intraexaminador quando comparado com o interexaminador é esperado, demonstrando a confiabilidade na interpretação dos casos.

\section{DISCUSSÃO}

Alguns autores $3,7,13$ salientam a importância do estabelecimento da maturidade esquelética no diagnóstico ortodôntico, uma vez que esse não deve basear-se apenas na avaliação das estruturas e funções presentes, mas também numa estimativa de quando e quanto é a expectativa do crescimento craniofacial. O crescimento fisiológico pode auxiliar o tratamento das más oclusões, enquanto que sua ausência pode indicar a necessidade de tratamento ortodôntico-cirúrgico.

$\mathrm{Na}$ avaliação intraexaminador (Tab. 2) observouse que os escores atribuídos às telerradiografias concordaram em 80 dos 100 casos para o examinador $\mathrm{A}$, em 95 dos 100 casos para o examinador B e em 77 dos 100 casos para o examinador C. Os resultados da avaliação intraexaminador denotam que houve, portanto, uma quantidade relativamente pequena de variação nos resultados em duas etapas ou, de outra forma, que houve uma reprodutibilidade na interpretação dos casos.

Os resultados da comparação interexaminadores demonstraram que houve uma concordância de classificação de 76 telerradiografias para os testes $\mathrm{A} \times \mathrm{B}$ e $\mathrm{A} \times \mathrm{C}$ e de 75 telerradiografias para o teste $\mathrm{B} \times \mathrm{C}$.

$\mathrm{Na}$ grande maioria das comparações, tanto intra como interexaminadores, os resultados não coincidentes variaram apenas um escore, por exemplo, enquanto o examinador A atribuiu o escore de Iniciação para a telerradiografia número 12 , o examinador $\mathrm{B}$ atribuiu o escore subseqüente (Aceleração) para a mesma telerradiografia. De acordo com Hassel e Farman ${ }^{9}$, entretanto, essa flutuação entre dois escores contíguos não apresenta relevância clínica para invalidar o método, e esses resultados devem ser considerados como aceitáveis. Muitos casos dúbios podem não permitir a deter- minação de um estágio com precisão, principalmente se for considerado que a radiografia pode ter sido obtida quando estava ocorrendo a transição de um estágio para outro subseqüente. Assim, a avaliação pode ocorrer tanto no início quanto no final de um determinado estágio de maturação e o mais provável, nessas situações, é que ele assuma as características semelhantes aos estágios que o precedem ou o sucedem.

A utilização de papel cartão preto vedando tanto a identificação, quanto a idade, o gênero e os elementos dentários teve o intuito de eliminar possíveis influências destes fatores durante a atribuição dos escores na avaliação das telerradiografias. Santos et al. ${ }^{18}$ relatam que a utilização de uma "máscara" de cartão preto nas demais estruturas da radiografia evitaria a atribuição de um escore tendencioso, principalmente nos casos mais confusos.

Com base nessas observações, concordamos com Santos, Almeida ${ }^{17}$ e Armond ${ }^{1}$ quando descrevem que as vértebras podem auxiliar na determinação do estágio de maturação óssea, no qual o indivíduo se encontra, mas não devem ser utilizadas de forma absoluta. Como qualquer outro método, torna-se possível complementá-lo com o maior número de informações disponíveis durante anamnese e exame físico do paciente ${ }^{10}$.

\section{CONCLUSÃO}

A partir da metodologia adotada e das considerações expostas, podemos concluir que:

- O método de determinação da maturação esquelética por meio das vértebras cervicais mostrouse reproduzível na avaliação do estágio em que o indivíduo se encontra na curva de crescimento. 


\title{
Evaluation of the reproducibility of the method of determination of the skeletal maturation by cervical vertebrae
}

\begin{abstract}
Among the different ways to determine the degree of the skeletal maturation of the patient, the Lamparski method stands out modified by Hassel e Farman that proposed the identification of the maturation stage throught anatomic changes of the $2^{\text {nd }}$, $3^{\text {rd }}$ and $4^{\text {th }}$ cervical vertebrae. Awere of the qualities conferred to the quoted method emerged the interest about evaluating its reprodutictibility with the aim of divulge and incorporate it as an element in diagnoses and as an auxiliary in the prognoses of the malocclusion treatment. The sample presented 100 teleradiographs in lateral norm of the patients examined for orthodontic treatment in School of Dentistry at Araçatuba - São Paulo state University in 2000 and 2001. Patients of both genders at the age of 6 through 16 years old and average age of 9 and 7 months in the sample. Three examiners properly trained did the evaluation of the teleradiographs rating them in phases from one to six. After analyzing the results, these ones were tabulated and subdwed to the KAPPA coefficient of agreement to evaluation inter and intra-examiner, concluding this way, the reproductibility of the above mentioned method. The method of the skeletal maturation by cervical vertebral was reproducible to determine the patient's stage in the curve of growth.
\end{abstract}

Key words: Skeletal age. Skeletal maturation indicators. Lateral cephalometric radiographs. Cervical vertebrae. Craniofacial growth.

\section{REFERÊNCIAS}

1. ARMOND, M. C. Estimativa do surto de crescimento puberal pela avaliação das vértebras cervicais em radiografias cefalométricas laterais. 2000. Dissertação (Mestrado) - Faculdade de Odontologia de São José dos Campos, Universidade Estadual Paulista "Júlio de Mesquita Filho", São José dos Campos, 2000.

2. BLAND, J. H. Disorders of cervical spine. Philadelphia: WB Saunders, 1987

3. BROADBENT, B. H.; GOLDEN, W. H. The value of an assessment of skeletal maturity in orthodontic diagnosis. Am J Phys Anthropol, Philadelphia, v. 34, no. 3, p. 409-410, 1971.

4. FISHMAN, L. S. Radiographic evaluation of skeletal maturation: a clinically oriented method based on hand-wrist films. Angle Orthod, Appleton, v. 52, no. 2, p. 88-112, Apr. 1982

5. GENEROSO, R. et al. Estudo da correlação entre a idade cronológica e a maturação das vértebras cervicais em pacientes em fase de crescimento puberal. R Dental Press Ortodon Ortop Facial, Maringá, v. 8, n. 4, p. 19-36, jul./ago. 2003.

6. GREULICH, W. W.; PYLE, S. I. Radiographic atlas of skeletal development of hand and wrist. 2nd ed. California: Standford University Press, 1949.

7. GUZZI, B. S. S.; CARVALHO, L. S. Estudo da maturação óssea em pacientes jovens de ambos os sexos através de radiografias de mão e punho. Ortodontia, São Paulo, v. 33, n. 3, p. 49-58, set./dez. 2000.

8. HÄGG, U.; TARANGER, J. Maturation indicators and puberal growth spurt. Am J Orthod, St. Louis, v. 82, no. 4, p. 299-309, Oct. 1982.

9. HASSEL, B.; FARMAN, A. G. Skeletal maturation evaluation using cervical vertebrae. Am J Orthod Dentofacial Orthop, St. Louis, v.107, no.1, p. 58-66, Jan. 1995

10. HOUSTON, W. J. B. Relationships between skeletal maturity estimated from hand-wrist radiographs and the timing of adolescent growth spurt. Eur J Orthod, London, v. 2, p. 81-93, 1980.

11. LAMPARSKI, D. G. Skeletal age assessment utilizing cervical vertebrae. Pittsburgh: University of Pittsburgh, 1972. Abstract.

12. LANDIS, J. R.; KOCH, G. G. The measurement of observer agreement for categorical data. Biometrics, Washington, DC, v. 33, p.159-174, 1977

13. MARTINS, J. C. R.; SAKIMA, T. Considerações sobre a previsão do surto de crescimento puberal. Ortodontia, São Paulo, v. 10, n. 3, p.164-170, dez. 1977

14. MERCADANTI, M. M. N. Radiografia de mão e punho. In: FERREIRA, F. V. Ortodontia: diagnóstico e planejamento clínico. 4. ed. São Paulo: Artes Médicas, 2001. p.187-223

15. MORAES, M. E. L. Surto de crescimento puberal: relação entre mineralização dentária, idade cronológica, idade dentária e idade óssea: método radiográfico. 1997. Tese (Doutora- do em Radiologia Odontológica) - Faculdade de Odontologia de São José dos Campos, Universidade Estadual Paulista "Júlio de Mesquita Filho", São José dos Campos, 1997.

16. ROSA, J. E. Legislação e normas do emprego de raios-X. In: FREITAS, A.; ROSA, J. E.; SOUZA, I. F. Radiologia odontológica. 4. ed. São Paulo: Artes médicas, 1998. p. 687-699.

17. SANTOS, S. C. N.; ALMEIDA, R. R. Estudo comparativo de dois métodos de avaliação da idade esquelética utilizando telerradiografias em norma lateral e radiografias carpais. Ortodontia São Paulo, v. 32, n. 2, p. 33-44, maio/ago. 1999.

18. SANTOS, S. C. N.; ALMEIDA, R. R.; HENRIQUES, J. F. C.; BER TOZ, F. A.; ALMEIDA, R. R. Avaliação de um método de determinação do estágio de maturação esquelética utilizando as vértebras cervicais presentes nas telerradiografias em norma lateral. R Dental Press Ortodon Ortop Facial, Maringá, v. 3, n. 3, p. 67-77, maio/jun. 1998

19. SANTOS, E. C. A.; HENRIQUES, J. F. C. Estudo cefalométrico entre as alterações resultantes de dois tipos de tratamento da má oclusão de Classe II, 1a divisão, de Angle. R Dental Press Ortodon Ortop Facial, Maringá, v. 5, n. 6, p.11-31, nov./dez. 2000.

20. SILVA FILHO, O. G; SAMPAIO, L. L.; FREITAS, J. A. S. Avaliação de um método simplificado para estimar a maturação esquelética. Ortodontia, São Paulo, v. 25, n. 1, p. 21-35, jan./abr. 1992.

21. TULLOCH, J. F. C.; PROFFIT, W. R.; PHILLIPS, C. Benefit of early Class II treatment: progress report of a two-phase randomized clinical trial. Am J Orthod Dentofacial Orthop, St. Louis, v. 113 , no. 1 , p. 62-72, Jan. 1998

22. TULLOCH, J. F. C.; PROFFIT, W. R.; PHILLIPS, C. Influences on the outcome of early treatment for Class II malocclusion. Am J Orthod Dentofacial Orthop, St. Louis, v. 111, no. 5, p. 533542, May 1997.

23. URSI, W. J. S. Determinação da maturidade esquelética através de radiografias carpais: sua importância no tratamento ortodôntico-ortopédico. In: INTERLANDI, S. Ortodontia: bases para Iniciação. 3. ed. São Paulo: Artes Médicas, 1999. p. 377-391.

\section{Endereço para correspondência}

Eduardo César Almada Santos

Disciplina de Ortodontia

Faculdade de Odontologia de Araçatuba - UNESP

Rua José Bonifácio, 1193

Araçatuba - São Paulo

CEP - 16015-050

E-mail: almada@foa.unesp.br 